\title{
Enhanced spectral resolution in immobilized peptides and proteins by combining chemical shift sum and difference spectroscopy ${ }^{\text {is }}$
}

\author{
Sorin Luca and Marc Baldus* \\ Department for NMR-based Structural Biology, Max-Planck-Institute for Biophysical Chemistry, Solid-state NMR, Am Fassberg 11, \\ 37077 Göttingen, Germany
}

Received 7 February 2002; revised 13 September 2002

\begin{abstract}
A two-dimensional correlation experiment is introduced that records the sum and difference chemical shift of two scalar or dipolar coupled nuclei. Statistical results indicate that the suggested pulse scheme can significantly increase the possibility of separating chemical shift contributions due to residue type and backbone conformation in immobilized peptides and proteins. Experimental applications demonstrate the theoretical concept and lead to the predicted resolution enhancement between different amino acid types and among protein residues of different secondary structure.
\end{abstract}

(C) 2002 Elsevier Science (USA). All rights reserved.

Keywords: Chemical shifts; Correlation spectroscopy; Magic angle spinning; Protein backbone structure; Spectral resolution

\section{Introduction}

Whenever Nuclear Magnetic Resonance (NMR) spectroscopy is utilized for the characterization of multiple-labelled peptides and proteins, the observed spectral dispersion can be significantly influenced by conformation-dependent chemical shift contributions under solution-state (see e.g., [1-4]) or solid-phase (see e.g., [5-7]) conditions. In the solid-state, these effects can e.g., be observed under high-resolution magic angle spinning [8] where recent advancements in solid-state NMR methodology and sample preparation have allowed for full or at least partial resonance assignments of multiply labelled proteins [9-12] and a membrane protein complex [13]. In extreme cases, a small number of residue types may contribute to the protein primary sequence and spectral dispersion can depend critically on multi-dimensional schemes that are susceptible to changes in backbone or side chain conformation. At the same time, the sensitivity of the NMR method of choice should be sufficient to allow for structural studies in a

\footnotetext{
Presented in part at the 43rd ENC conference, Asilomar, CA, April 14th-19th, 2002.

${ }^{*}$ Corresponding author. Fax: (+49)-551201-2212/2202.

E-mail address: maba@nmr.mpibpc.mpg.de (M. Baldus).
}

variety of immobilized polypeptides including protein aggregates or membrane proteins.

The relationship between protein secondary structure and the observed isotropic chemical shift has been investigated experimentally $[1-4,14]$ and theoretically [14-16] for a long time. Empirical solution state studies have, for example, found a remarkable correlation between $C_{\mathrm{A}}$ and $C_{\mathrm{B}}$ isotropic chemical shift values and protein secondary structure [4]. Likewise, the calculated difference between the experimentally observed $C_{\mathrm{A}}$ and $C_{\mathrm{B}}$ isotropic chemical shift values has been correlated with protein backbone structure in solution and solidphase applications [7,17]. Alternatively, one can examine whether the sum of the two chemical shift values readily obtained from the evolution dimension of a double-quantum correlation experiment $[18,19]$, may serve as a qualitative indicator of protein backbone conformation. To investigate the correlation between these experimentally detected parameters and the local protein topology on a broader basis, we have analysed an empirical database containing chemical shift values of 40 globular proteins studied by solution-state NMR methods [20]. For simplicity, we only consider protein regions of defined backbone conformation (conf) and we follow the general assumption that chemical shift contributions due to amino acid type, backbone 
conformation, hydrogen bonding, or local charge interactions are additive [16]. For each of the resonance frequencies and observed experimentally the parameters $\Omega_{\mathrm{CA}}$ and $\Omega_{\mathrm{CB}}$ :

$\Delta \bar{\Omega}(\operatorname{conf})=\frac{1}{N_{\text {conf }}} \sum_{n=1}^{N_{\text {conf }}} \Omega_{\mathrm{CA}}(n)-\Omega_{\mathrm{CB}}(n)$,

$\Sigma \bar{\Omega}($ conf $)=\frac{1}{N_{\text {conf }}} \sum_{n=1}^{N_{\text {conf }}} \Omega_{\mathrm{CA}}(n)+\Omega_{\mathrm{CB}}(n)$,

represent a qualitative measure for conformationdependent chemical shift contributions (averaged over $N_{\text {conf }}$ database entries in a given conformation) for a particular residue type. To simplify the analysis, we focus here on $\alpha$-helical (i.e., $\operatorname{conf}=\mathrm{H}$ ) and $\beta$-sheet (denoted by $\operatorname{conf}=\mathrm{E}$ ) regions of the investigated proteins. The spectral dispersion between the two conformations considered can be mathematically related to the parameters

$\Delta_{\mathrm{H}, \mathrm{E}}=\Delta \bar{\Omega}(\mathrm{H})-\Delta \bar{\Omega}(\mathrm{E})$,

$\Sigma_{\mathrm{H}, \mathrm{E}}=\Sigma \bar{\Omega}(\mathrm{H})-\Sigma \bar{\Omega}(\mathrm{E})$,

that are depicted in Fig. 1 for all relevant residue types. Except for the unique residues glycine and proline, $\Delta_{\mathrm{H}, \mathrm{E}}$ exhibit values from $5 \mathrm{ppm}$ (cysteine) to more than $8 \mathrm{ppm}$ (alanine and valine). On the other hand, the parameter $\Sigma_{\mathrm{H}, \mathrm{E}}$ is significantly smaller for the majority of the considered residue types. Relative to $\Sigma_{\mathrm{H}, \mathrm{E}}, \Delta_{\mathrm{H}, \mathrm{E}}$ increases from a factor of two for histidine, serine, and threonine residues to more than 16 in the case of alanine. In agreement with the solid-state data discussed in [7] one may hence expect an increase in the spectral dispersion within each protein residue type if an NMR experiment is devised that encodes the chemical shift difference of two dipolar or scalar coupled spins in one spectral dimension. On the other hand, Fig. 1 suggests that the parameter $\Sigma_{\mathrm{H}, \mathrm{E}}$ in many cases reduces chemical shift variations due to changes in local backbone structure and it may hence offer the possibility for separation of different amino acid types with increased reliability during the NMR experiment.

For most amino acids, conformation-dependent changes of $C_{\mathrm{B}}$ resonances alone are comparable in size to deviations involving $C_{\mathrm{A}}$ chemical shifts. The increased size of the $\Delta_{\mathrm{H}, \mathrm{E}}$ parameters is hence caused by an additional sign change from $C_{\mathrm{A}}$ to $C_{\mathrm{B}}$ secondary chemical shifts for $\alpha$-helical and $\beta$-sheet conformations. Both contributions enter $\Delta_{\mathrm{H}, \mathrm{E}}$ in an additive manner and are included in Fig. 1 as grey $\left(C_{\mathrm{A}}\right)$ and dark grey $\left(C_{\mathrm{B}}\right)$ parts.

\section{Results and discussion}

A correlation experiment that encodes the sum and difference chemical shift frequency (in the following termed S/D experiment) between two dipolar or scalar

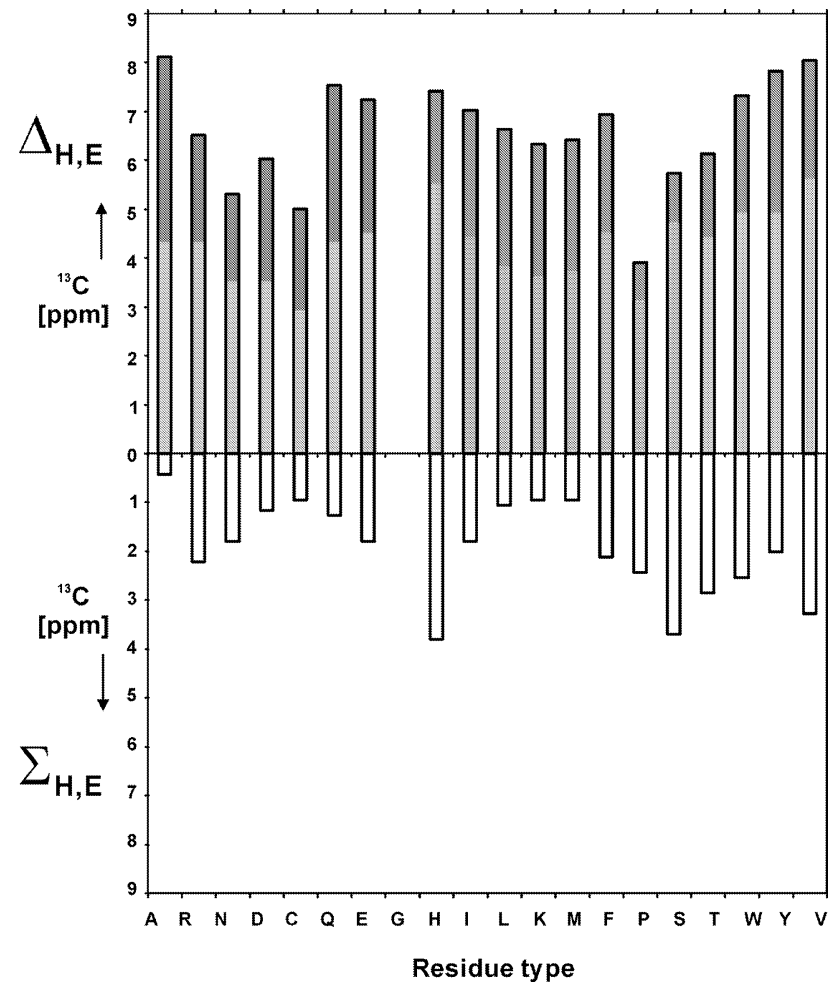

Fig. 1. Histogram correlating the parameters $\Delta_{\mathrm{H}, \mathrm{E}}=\Delta \bar{\Omega}(\mathrm{H})-\Delta \bar{\Omega}(\mathrm{E})$ and $\Sigma_{\mathrm{H}, \mathrm{E}}=\Sigma \bar{\Omega}(\mathrm{H})-\Sigma \bar{\Omega}(\mathrm{E})$ (see Eqs. (1) and (2)) with the protein residue type. The statistical data obtained from Iwadate et al. [20] were analyzed in the following way: first, all protein residues in $\alpha$-helical and $\beta$-sheet conformations were collected. Subsequently, the average difference $\Delta_{\mathrm{H}, \mathrm{E}}$ (top) and sum $\Sigma_{\mathrm{H}, \mathrm{E}}$ (bottom) chemical shift was computed for the two considered backbone conformations. Indicated are average chemical shift values (see Eq. (1)) with standard deviations ranging between 1 and $2 \mathrm{ppm}$ for all residue types. Individual average chemical shift contributions of $C_{\mathrm{A}}$ (grey) and $C_{\mathrm{B}}$ (dark grey) are included (top) for comparison. Protein residues are given in single letter notation. Finally, we give the number of data base entries corresponding to helical and sheet conformations, respectively, for each protein residue type: A $\left(N_{\mathrm{H}}=100, N_{\mathrm{E}}=42\right), \mathrm{R}(52,28), \mathrm{N}(32,16), \mathrm{D}(35,23), \mathrm{C}$ (11,16), Q (41,21), E (100,54), G (0,0), H $(15,8)$, I $(48,78), \mathrm{L}(93,74), \mathrm{K}$ (77,55), M (27,18), F (48,45), P (13,12), S (30,42), T $(39,77)$, W $(12,13)$, $\mathrm{Y}(26,36), \mathrm{V}(56,96)$.

coupled nuclei can in principle be obtained by combining a double-quantum (2Q) and zero-quantum (0Q) evolution dimension. Both types of transfer schemes have been successfully demonstrated in solution $[18,21]$ and solid-phase $[19,22,23]$ applications. We here suggest an alternative two-dimensional correlation experiment that contains a concerted set of two evolution dimensions (Fig. 2). Conventional cross polarization from ${ }^{1} \mathrm{H}$ to ${ }^{13} \mathrm{C}$ nuclei creates initial carbon polarization described by the density operator

$\sigma\left(t_{1 \mathrm{a}}=0\right)=\sum_{i} I_{i x}$.

After the first evolution period the density matrix reads, under the influence of the isotropic chemical shifts $\Omega_{i}$, as 


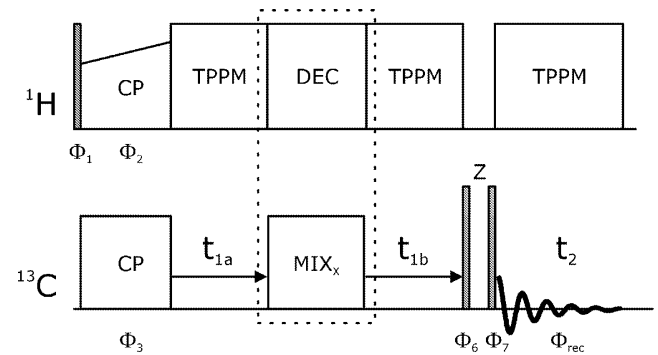

(a)

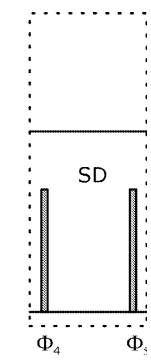

(b)
Fig. 2. (a) NMR pulse scheme to encode the sum and difference chemical shift frequency between two dipolar or scalar coupled homonuclear nuclei (S/D experiment). Both parameters determine the peak position in the indirect dimension of a conventional twodimensional experiment. After an amplitude modulated [40,41] Hartmann-Hahn cross polarization $[42,43]$ step, two synchronized evolution periods $\left(t_{1 \mathrm{a}}\right.$ and $\left.t_{1 \mathrm{~b}}\right)$ are separated by a mixing unit. In the current context, a proton-driven spin diffusion (SD) block [30] is utilized (b). The $z$-filter [24] at the end of the evolution period removes undesired coherences. TPPM decoupling [44] is used on the ${ }^{1} \mathrm{H}$ channel during free evolution periods. Indicated r.f. and receiver phases are given in detail in the text. Depending on the $\left({ }^{13} \mathrm{C},{ }^{13} \mathrm{C}\right)$ polarization transfer scheme, appropriate proton decoupling (DEC) during mixing can be applied.

$$
\sigma\left(t_{1 \mathrm{a}}\right)=\sum_{i} I_{i x} \cos \left(\Omega_{i} t_{\mathrm{la}}\right)+\sum_{i} I_{i y} \sin \left(\Omega_{i} t_{\mathrm{la}}\right) .
$$

Setting $\phi_{4}=\phi_{3}+\pi / 2$ (see Fig. 2) at the beginning of the mixing period allows for the selection of the component along the $x$-axis of the rotating frame in Eq. (4). During mixing, this component further evolves to

$$
\begin{aligned}
\sigma_{x}\left(t_{\mathrm{lb}}=0\right)= & \sum_{i} d_{i x, i x}\left(t_{\text {mix }}\right) \cos \left(\Omega_{i} t_{\mathrm{la}}\right) I_{i x} \\
& +\sum_{\substack{i, j \\
i \neq j}} d_{i x, j x}\left(t_{\text {mix }}\right) \cos \left(\Omega_{i} t_{\mathrm{la}}\right) I_{j x} .
\end{aligned}
$$

The amplitudes $d_{i x, i x}\left(t_{\text {mix }}\right)$ and $d_{i x, j x}\left(t_{\text {mix }}\right)$ reflect the general transfer characteristics of the mixing unit and $\Omega_{i, j}$ refer to the isotropic chemical shift values of spin $i$ and $j$, respectively. Contributions along the $x$-axis of the rotating frame are, at the end of the second evolution block, described by

$$
\begin{aligned}
\sigma_{x}\left(t_{\mathrm{lb}}\right)= & \sum_{i} d_{i x, i x}\left(t_{\text {mix }}\right) \cos \left(\Omega_{i} t_{\mathrm{la}}\right) \cos \left(\Omega_{i} t_{\mathrm{lb}}\right) I_{i x} \\
& +\sum_{\substack{i, j \\
i \neq j}} d_{i x, j x}\left(t_{\mathrm{mix}}\right) \cos \left(\Omega_{i} t_{1 \mathrm{a}}\right) \cos \left(\Omega_{j} t_{\mathrm{lb}}\right) I_{j x} .
\end{aligned}
$$

A $z$-filter [24] and appropriate phase cycling ensures that only the transfer amplitudes given in Eq. (6) are selected. For example, for longitudinal mixing indicated in Fig. 2 we can utilize $\phi_{1}=\{+y\}_{4},\{-y\}_{4} ; \phi_{2}=+x$; $\phi_{3}=+x ; \quad \phi_{4}=+y ; \quad \phi_{5}=-y ; \quad \phi_{6}=+y ; \quad \phi_{7}=\{-y$, $+x,+y,-x\} ; \quad \phi_{\mathrm{rec}}=\{+x,+y,-x,-y-x,-y,+x,+y\}$ (see Fig. 2 for notation). Conventional quadrature detection guarantees phase sensitive detection in $t_{2}$, while TPPI phase cycling [25] can be employed to construct a complex signal amplitude along $t_{1}$. For this purpose, the $\mathrm{CP}$ phase $\phi_{3}$ is incremented by $90^{\circ}$ between subsequent experiments.

Assuming $t_{1 \mathrm{a}}=t_{1 \mathrm{~b}}$, the second term in Eq. (6) can be mathematically rearranged to contain the desired correlations $\Omega_{i} \pm \Omega_{j}$. The observed signal intensities depend on the type of the mixing sequence and the length of the mixing time. Since the S/D experiment is applied in homonuclear spin systems, auto correlation peaks in the two-dimensional pattern occur along the $\left(0, \Omega_{i}\right)$ and $\left(2 \Omega_{i}, \Omega_{i}\right)$ frequency axes due to polarization that is not transferred during the mixing unit (first term in Eq. (6)). These correlation patterns can be minimized if one utilizes scalar through-bond transfer schemes (see e.g., $[26,27])$ or adiabatic through-space transfer mechanisms [27-29]. Both transfer mechanisms also maximize the desired transfer amplitudes observed at the frequency positions $\left(\Omega_{i} \pm \Omega_{j}, \Omega_{i, j}\right)$. To demonstrate the experimental concept, we here utilized a conventional ${ }^{13} \mathrm{C}$ proton-mediated spin diffusion unit [30]. We also note that a concerted set of evolution dimensions such as shown in Fig. 2 has previously been shown to reduce the dimensionality of a triple-resonance assignment experiment in solution-state [31,32] or solid-phase systems [33].

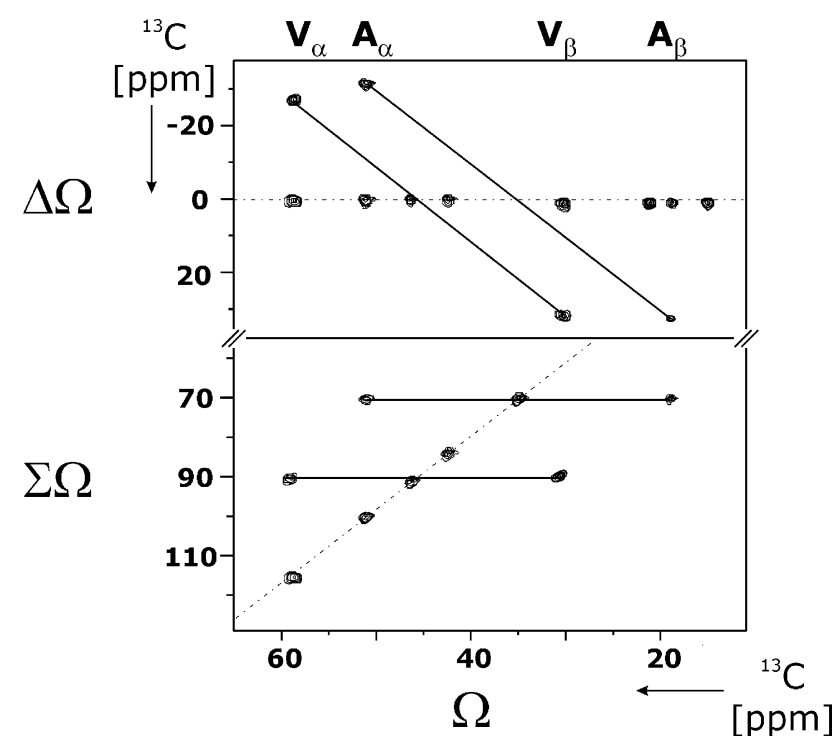

Fig. 3. S/D correlation experiment in a mixture of $\mathrm{U}-\left[{ }^{13} \mathrm{C},{ }^{15} \mathrm{~N}\right]$ labelled variants of the tri-peptide Ala-Gly-Gly $(10 \mathrm{mg})$ and FMOC-Valine $(10 \mathrm{mg})$. Except for the two sets of auto correlation peaks (dotted lines), the aliphatic spectrum only contains the desired correlations of the type shown on top and shown at the bottom. In total, $256 t_{1}$ experiments were recorded using a homonuclear spin diffusion time of $20 \mathrm{~ms}$. In $t_{2}, 16$ transients were averaged giving rise to a total acquisition time of $2.3 \mathrm{~h}$. In both dimensions, a dwell time of $10 \mu \mathrm{s}$ was used. Before complex Fourier transformation, the recorded data were zerofilled to a $1024 \times 1024$ data matrix. MAS rate and temperature were actively controlled at $11 \mathrm{kHz}$ and $4{ }^{\circ} \mathrm{C}$, respectively. The S/D experiment was conducted on a wide-bore $400 \mathrm{MHz}$ NMR instrument (Bruker/Germany). 
In Fig. 3 we present results of an S/D correlation experiment in a mixture of $\mathrm{U}-\left[{ }^{13} \mathrm{C},{ }^{15} \mathrm{~N}\right]$ labelled variants of the tri-peptide Ala-Gly-Gly and FMOC-Valine. Except for the two sets of auto correlation peaks, the S/ D-NMR spectrum in the side chain regime only contains the desired correlations of the type (Fig. 3, top) and (Fig. 3, bottom), respectively. In general, the transfer amplitudes represent the polarization transfer pathways $C_{\mathrm{A}} \rightarrow C_{\mathrm{B}}$ and $C_{\mathrm{A}} \leftarrow C_{\mathrm{B}}$. For the segment containing the chemical shift difference pattern one hence observes a sign change from $\Delta \Omega=\Omega_{\mathrm{CA}}-\Omega_{\mathrm{CB}}$ to $-\Delta \Omega=\Omega_{\mathrm{CB}}-$ $\Omega_{\mathrm{CA}}$ in the indirect dimension. Only the correlations involving $\Sigma \Omega$ are explicitly dependent on the selected r.f. carrier frequency and they can hence be placed outside the chemical shift difference pattern if necessary. In Fig. 3 , the larger dispersion is obtained in the spectral segment containing the $\Sigma \Omega$ parameters in the indirect dimension. The spectral dispersion is predominantly given by the amino acid type and conformation dependent chemical shift contributions are here of minor relevance.

In contrast, the spectral dispersion among residues of a particular amino acid type can significantly increase using information obtained in the chemical shift difference segment of the S/D experiment. For example, we analyse in Fig. 4 experimental results obtained on an immobilized $\mathrm{U}-\left[{ }^{13} \mathrm{C},{ }^{15} \mathrm{~N}\right]$ labelled version of ubiquitin for which partial resonance assignments in the solidstate have previously been reported [9,34]. In Fig. 4 the sum $(\Sigma \Omega$ in a) and difference $(\Delta \Omega$ in b) segments of the $\mathrm{S} / \mathrm{D}$ correlation spectrum are shown. Again, the sum chemical shift spectrum is identical to a conventional $2 \mathrm{Q}$ correlation experiment along the $(2 \Omega, \Omega)$ diagonal while the spectral region containing the difference spectrum reflects a $0 \mathrm{Q}$ correlation pattern centred at the $(0, \Omega)$ axis. Since ubiquitin exhibits very similar structures in the solid-phase $[9,34,35]$ and solution-state [36], we can analyse the S/D spectrum using solution-state chemical shift predictions for the resolved ${ }^{13} \mathrm{C}$ resonances. The resulting NMR assignments in the solid-state are summarized in Table 1 together with backbone dihedral angles as obtained from solution-state NMR.

In Figs. $4 \mathrm{c}$ and $\mathrm{d}$, we focus on the (well separated) spectral region containing correlations due to the seven threonine residues found in ubiquitin. Since we are now considering a particular residue type, the spectral dispersion in the indirect dimension should be predominantly given by variations in the local backbone conformation. In the case of threonine, three groups of

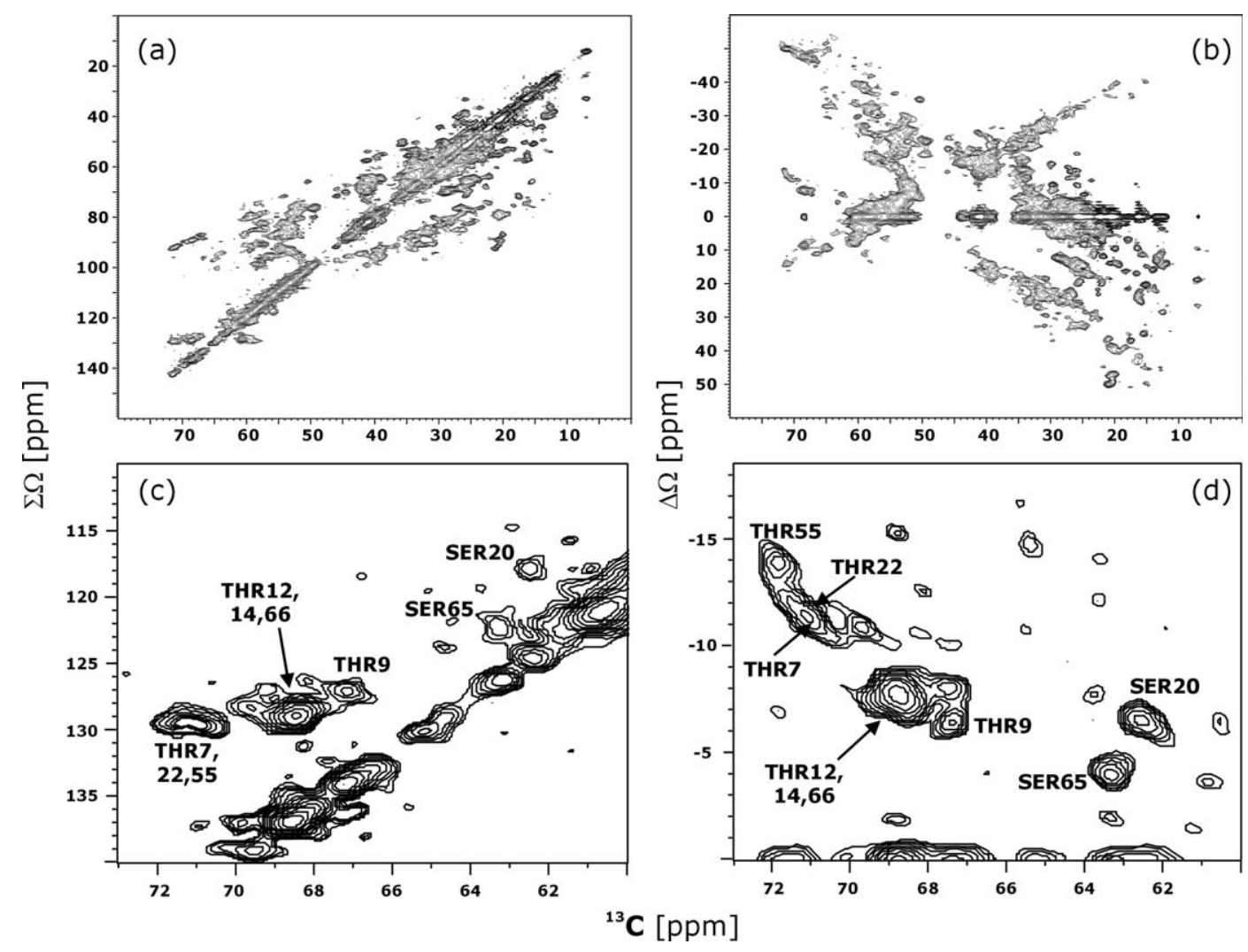

Fig. 4. Experimental sections of an S/D correlation experiment conducted on a $5 \mathrm{mg}$ sample of $\mathrm{U}_{-}\left[{ }^{13} \mathrm{C},{ }^{15} \mathrm{~N}\right]$ labelled ubiquitin (obtained from VLI research, Malvern, PA). Displayed are the sum $\Sigma \Omega$ (a) and difference $\Delta \Omega$ (b) chemical shift spectrum in the side chain region of the protein. Expanded sections that contain threonine and serine correlations of the type $\left(\Sigma \Omega, \Omega_{\mathrm{CA} . \mathrm{CB}}\right)$ (c) and $\left(\Delta \Omega, \Omega_{\mathrm{CA} \text {.СB }}\right)(\mathrm{d})$ are included. Here, $736 t_{1}$ experiments were recorded using 128 transients in $t_{2}$ on a WB $400 \mathrm{MHz}$ instrument (total measurement time: $53 \mathrm{~h}$ ). Again a homonuclear mixing time of $20 \mathrm{~ms}$ was employed using an MAS rate of $11 \mathrm{kHz}$ at $-7^{\circ} \mathrm{C}$. For the sake of clarity, the spectra (a) and (b) were plotted using a smaller contour level linewidth. 
Table 1

Tentative solid-state NMR assignments of ubiquitin from the S/D spectrum shown in Fig. 4

\begin{tabular}{|c|c|c|c|c|c|c|}
\hline \multicolumn{2}{|c|}{ Residue } & \multicolumn{2}{|c|}{${ }^{13} \mathrm{C}$ chemical shift (ppm) } & \multicolumn{3}{|c|}{ Torsion angles } \\
\hline Type & Number & $\Sigma \Omega$ & $\Delta \Omega$ & $\psi$ & $\phi$ & conf \\
\hline ALA & 28 & 72 & 37 & -38.1 & -66.1 & $\mathrm{H}$ \\
\hline \multirow[t]{2}{*}{ ASN } & 25 & 92 & 18 & -44.4 & -65.5 & $\mathrm{H}$ \\
\hline & 60 & 91 & 16 & 45.4 & 57.9 & $\mathrm{~T}$ \\
\hline \multirow[t]{4}{*}{ ILE } & 3 & 99 & 16 & 163.0 & -131.1 & $\mathrm{E}$ \\
\hline & 13 & 99 & 18.5 & 142.0 & -109.5 & $\mathrm{E}$ \\
\hline & 23 & 97 & 28.5 & -37.2 & -61.3 & $\mathrm{H}$ \\
\hline & 30 & 100 & 29 & -39.6 & -70.0 & $\mathrm{H}$ \\
\hline LEU & 15 & 97 & 6 & 154.0 & -126.4 & $\mathrm{E}$ \\
\hline PRO & 38 & 97 & 32.5 & -32.2 & -57.2 & G \\
\hline \multirow[t]{2}{*}{ SER } & 20 & 118 & -6.5 & -8.1 & -79.8 & $\mathrm{~T}$ \\
\hline & 65 & 122 & -4 & 159.5 & -71.1 & $\mathrm{~L}$ \\
\hline \multirow[t]{7}{*}{ THR } & 7 & 129.5 & -11 & 170.8 & -99.6 & $\mathrm{E}$ \\
\hline & 9 & 127 & -6 & 14.9 & -101.4 & $\mathrm{~T}$ \\
\hline & 12 & 129 & -7.5 & 131.8 & -119.9 & $\mathrm{E}$ \\
\hline & 14 & 129 & -7.5 & 139.7 & -101.4 & $\mathrm{E}$ \\
\hline & 22 & 129.5 & -11.5 & 160.4 & -83.7 & B \\
\hline & 55 & 129.5 & -14 & 164.6 & -104.5 & B \\
\hline & 66 & 129 & -7.5 & 126.7 & -119.2 & $\mathrm{E}$ \\
\hline VAL & 5 & 93.5 & 27 & 114.2 & -118.0 & $\mathrm{E}$ \\
\hline
\end{tabular}

Indicated are sum and difference chemical shifts $\Sigma \Omega=\Omega_{\mathrm{CA}}+\Omega_{\mathrm{CB}}$ and $\Delta \Omega=\Omega_{\mathrm{CA}}-\Omega_{\mathrm{CB}}$ (recorded in the S/D experiment) that can be utilized to calculate the chemical shifts of $C_{\mathrm{A}}$ and $C_{\mathrm{B}}$ nuclei, individually. In addition, dihedral angles as obtained from solution-state NMR (PDB entry 1D3Z) along with a one letter notation for the actual secondary structure conformation $(\mathrm{H}, \alpha$-helix; $\mathrm{B}$, isolated beta-bridge; $\mathrm{E}, \beta$-sheet; $\mathrm{T}$, hydrogen bonded turn; G, 3/10 helix; L, loop) are given.

residues (characterized by the dihedral angles given in Table 1) can be distinguished: (1) Thr 7, 22, and 55, (2) Thr 9, and (3) Thr 12, 14, and 66. A comparison of the sum (Fig. 4c) and difference (Fig. 4d) correlation spectra reveals that these groups are spread over more than $9 \mathrm{ppm}$ in the indirect $\Delta \Omega$ dimension (Fig. $4 \mathrm{~d}$ ). As a result, a tentative assignment of the signal set (Thr 7, 9, 22, and 55) can easily be obtained. In contrast, the total dispersion among threonine residues in the indirect $\Sigma \Omega$ dimension does not exceed $3 \mathrm{ppm}$ and a further spectral analysis remains difficult (Fig. 4c).

In agreement with the statistical analysis of Fig. 1, the difference correlation data hence can improve the spectral resolution if the backbone conformation within a particular protein residue type is variable. In Table 1, we have examined these dependencies for additional protein residues detectable in Fig. 4. In general, one finds that the spectral dispersion in the sum chemical shift segment $(\Sigma \Omega)$ of the $\mathrm{S} / \mathrm{D}$ spectrum is comparable or larger than in the $\Delta \Omega$ segment if (a) different amino acid types or (b) residues described by similar backbone conformations are to be studied. In contrast, the difference chemical shift spectrum $\Delta \Omega$ enhances the spectral dispersion within a particular residue type for variable backbone conformations in the polypeptide. In addition to the threonine signal set discussed above, the latter aspect is also observable for the residue types isoleucine and valine that are found in both $\alpha$-helical and $\beta$-sheet conformations in ubiquitin. The spectral dispersion in the $\Delta \Omega$ segment of the indirect dimension amounts to 13 and $10 \mathrm{ppm}$ among identified isoleucine and valine residues, respectively, exceeding the dispersion in a conventional double-quantum evolution dimension (see Table 1) by a factor $2-4$. In all cases, the size of $\Delta \Omega$ reflects qualitative information about the local backbone conformation: larger values correspond to helical conformations, while smaller values relate to extended regions of the protein. 
The experimental results obtained on ubiquitin indicate that even small variations in backbone conformation may suffice to disperse protein signals in a S/D two-dimensional ${ }^{13} \mathrm{C},{ }^{13} \mathrm{C}$ correlation experiment. In our applications, signal losses during the longer evolution time and the $z$-filter are negligible and the sensitivity of the $\mathrm{S} / \mathrm{D}$ experiment is comparable to a conventional 1Q-1Q correlation experiment assuming the same mixing scheme. In cases where amino acid type and backbone conformation are both similar, additional resolution might be obtained from an investigation of the side chain correlations, possibly by extending the method to three spectral dimensions or from further inter-residue correlation experiments [27].

\section{Conclusions}

We have introduced a two-dimensional correlation experiment that allows for the simultaneous detection of the sum and the difference in the chemical shift of two dipolar or scalar coupled spins under MAS conditions. An empirical analysis of $C_{\mathrm{A}}$ and $C_{\mathrm{B}}$ chemical shifts obtained in the solution and solid-phase indicates that the combination of both parameters can significantly enhance the possibilities for assignment and spectral dispersion of NMR signals of peptides and proteins. Our analysis reveals that in contrast to conventional 2Q spectroscopy, chemical shift difference correlations under MAS can result in a significant increase in spectral dispersion between protein residues of variable secondary structure. On the other hand, 2Q type correlations are preferable if variations in amino acid type or residues of similar backbone conformation are to be expected. The proposed concept directly delivers sum and difference chemical shift spectra during acquisition and hence does not require $0 \mathrm{Q} / 2 \mathrm{Q}$ filtering methods or a reprocessing of the detected data set [37]. Since singlequantum coherences are evolving, the linewidth is in both spectral dimensions given by the normal transverse $1 \mathrm{Q}$ relaxation rate. For each amino acid type, the S/D spectrum may hence directly encode qualitative information about the backbone conformation in a protein of unknown structure. Similar correlations have recently been discussed in the context of macroscopically oriented peptides [38] and they may facilitate the determination of structural constraints whenever two or higher dimensional correlation spectroscopy is utilized. In the context of ligand-binding interactions in membrane proteins or in other biophysical applications where unwanted background signals can be present, the S/D experiment can easily be extended to a multiple-quantum correlation experiment. Moreover, scalar or adiabatic mixing schemes can be employed to enhance the general sensitivity of the technique. Finally, proton-mediated polarization transfer schemes [39] can be incorporated to encode through-space $\left({ }^{1} \mathrm{H},{ }^{1} \mathrm{H}\right)$ contacts along the polypeptide chain in high spectral resolution.

We expect the current method to be of particular interest in cases where spectral overlap, due to e.g., protein size, primary sequence, or sample heterogeneity limits the applicability of other correlation experiments. Biophysical applications could include membrane protein ligands, protein aggregates or partially folded proteins in the solid-phase or in the solution-state. Our results obtained on a $400 \mathrm{MHz}$ NMR instrument suggest that the resulting two-dimensional correlation spectra might be sensitive to even small variations in backbone structure, in particular when studied under ultra-high magnetic field conditions. For applications in helical membrane proteins, extensions to three spectral dimensions that include additional side chain correlations, the incorporation of heteronuclear $\left({ }^{15} \mathrm{~N},{ }^{13} \mathrm{C}\right)$ correlation methods or the study of macroscopically oriented samples could further enhance the spectral resolution. A preliminary analysis of other protein resonances including ${ }^{1} \mathrm{H}$ and ${ }^{15} \mathrm{~N}$ nuclei indicates that the proposed experimental scheme might also be helpful for the characterization of other peptide and protein segments when studied under MAS conditions.

\section{Acknowledgments}

Discussions with Dr. Astrid Eijkelenboom, Dr. Markus Zweckstetter and, Prof. Christian Griesinger are gratefully acknowledged.

\section{References}

[1] H. Sternlicht, D. Wilson, Magnetic resonance studies of macromolecules. I aromatic-methyl interactions and helical structure effects in lysozyme, Biochemistry 6 (1967) 2881-2892.

[2] J.L. Markley, D.H. Meadows, O. Jardetzky, Nuclear magnetic resonance studies of helix-coil transitions in polyamino acids, $\mathrm{J}$ Mol. Biol. 27 (1967) 25-40.

[3] K.H. Gross, H.R. Kalbitzer, Distribution of chemical-shifts in H1 nuclear magnetic-resonance spectra of proteins, J. Magn. Reson. 76 (1988) 87-99.

[4] S. Spera, A. Bax, Empirical correlation between protein backbone conformation and C-Alpha and C-Beta C-13 nuclear-magneticresonance chemical-shifts, J. Am. Chem. Soc. 113 (1991) 54905492.

[5] H. Saito, Conformation-dependent C-13 chemical-shifts - a new means of conformational characterization as obtained by highresolution solid-state C-13 NMR, Magn. Reson. Chem. 24 (1986) 835-852.

[6] H. Saito, S. Tuzi, A. Naito, Empirical versus nonempirical evaluation of secondary structure of fibrous and membrane proteins by solid-state NMR: a practical approach, Annu. Rep. NMR spectrosc. 36 (1998) 79-121.

[7] S. Luca, D.V. Filippov, J.H. van Boom, H. Oschkinat, H.J.M. de Groot, M. Baldus, Secondary chemical shifts in immobilized peptides and proteins: a qualitative basis for structure refinement under magic angle spinning, J. Biomol. NMR 20 (2001) 325-331. 
[8] E.R. Andrew, A. Bradbury, R.G. Eades, Nuclear magnetic resonance spectra from a crystal rotated at high speed, Nature 182 (1958) 1659.

[9] S.K. Straus, T. Bremi, R.R. Ernst, Experiments and strategies for the assignment of fully $\mathrm{C}-13 / \mathrm{N}-15$-labelled polypeptides by solid state NMR, J. Biomol. NMR 12 (1998) 39-50.

[10] M. Hong, Resonance assignment of C-13/N-15 labeled solid proteins by two- and three-dimensional magic-angle-spinning NMR, J. Biomol. NMR 15 (1999) 1-14.

[11] A. McDermott, T. Polenova, A. Bockmann, K.W. Zilm, E.K. Paulsen, R.W. Martin, G.T. Montelione, Partial NMR assignments for uniformly (C-13, N-15)-enriched BPTI in the solid state, J. Biomol. NMR 16 (2000) 209-219.

[12] J. Pauli, M. Baldus, B. van Rossum, H. de Groot, H. Oschkinat, Backbone and side-chain C-13 and N-15 signal assignments of the alpha-spectrin $\mathrm{SH} 3$ domain by magic angle spinning solid-state NMR at 17.6 tesla, Chembiochem 2 (2001) 272-281.

[13] T.A. Egorova-Zachernyuk, J. Hollander, N. Fraser, P. Gast, A.J. Hoff, R. Cogdell, H.J.M. de Groot, M. Baldus, Heteronuclear 2Dcorrelations in a uniformly [C-13, N-15] labeled membraneprotein complex at ultra-high magnetic fields, J. Biomol. NMR 19 (2001) 243-253.

[14] D.S. Wishart, B.D. Sykes, Chemical-shifts as a tool for structure determination, Methods Enzymol. 239 (1994) 363-392.

[15] A.C. de Dios, J.G. Pearson, E. Oldfield, Secondary and tertiary structural effects on protein $\mathrm{nmr}$ chemical-shifts - an abinitio approach, Science 260 (1993) 1491-1496.

[16] D.S. Wishart, D.A. Case, Use of chemical shifts in macromolecular structure determination, Methods Enzymol. 338 (2001) 3-34.

[17] G. Kozlov, I. Ekiel, N. Beglova, A. Yee, A. Dharamsi, A. Engel, N. Siddiqui, A. Nong, K. Gehring, Rapid fold and structure determination of the archaeal translation elongation factor 1 beta from Methanobacterium thermoautotrophicum, J. Biomol. NMR 17 (2000) 187-194.

[18] A. Bax, R. Freeman, S.P. Kempsell, Natural abundance C-13-C13 coupling observed via double- quantum coherence, J. Am. Chem. Soc. 102 (1980) 4849-4851.

[19] E.M. Menger, S. Vega, R.G. Griffin, Observation of carbon carbon connectivities in rotating solids, J. Am. Chem. Soc. 108 (1986) 2215-2218.

[20] M. Iwadate, T. Asakura, M.P. Williamson, C-alpha and C-beta carbon-13 chemical shifts in proteins from an empirical database, J. Biomol. NMR 13 (1999) 199-211.

[21] L. Müller, Mapping of spin-spin coupling via zero-quantum coherence, J. Magn. Reson. 59 (1984) 326-331.

[22] J.M. Koons, G.E. Pavlovskaya, A.A. Jones, P.T. Inglefield, Determination of interatomic distances by zero-quantum correlation spectroscopy under rotational-resonance conditions, J. Magn. Reson. 124 (1997) 499-502.

[23] T.P. Spaniol, A. Kubo, T. Terao, Resolution enhancement of magic-angle spinning NMR spectra for paramagnetic solids by zero-quantum NMR, Mol. Phys. 96 (1999) 827-834.

[24] R.R. Ernst, G. Bodenhausen, A. Wokaun, in: Principles of Nuclear Magnetic Resonance in One and Two Dimensions, Clarendon Press, Oxford, 1987.

[25] G. Bodenhausen, R.L. Vold, R.R. Vold, Multiple quantum spinecho spectroscopy, J. Magn. Reson. 37 (1980) 93-106.
[26] M. Baldus, B.H. Meier, Total correlation spectroscopy in the solid state. The use of scalar couplings to determine the through-bond connectivity, J. Magn. Reson. Ser. A 121 (1996) 65-69.

[27] M. Baldus, Correlation experiments for assignment and structure elucidation of immobilized polypeptides under magic angle spinning, Prog. Nucl. Magn. Reson. Spectrosc. 41 (2002) 1-47.

[28] R. Verel, M. Baldus, M. Nijman, J.W.M. van Os, B.H. Meier, Adiabatic homonuclear polarization transfer in magic-anglespinning solid-state NMR, Chem. Phys. Lett. 280 (1997) 31-39.

[29] R. Verel, M. Baldus, M. Ernst, B.H. Meier, A homonuclear spinpair filter for solid-state NMR based on adiabatic-passage techniques, Chem. Phys. Lett. 287 (1998) 421-428.

[30] N. Bloembergen, On the interaction of Nuclear Spins in a crystalline lattice, Physica 15 (1949) 386-426.

[31] T. Szyperski, G. Wider, J.H. Bushweller, K. Wüthrich, Reduced dimensionality in triple-resonance NMR experiments, J. Am. Chem. Soc. 115 (1993) 9307-9308.

[32] T. Szyperski, B. Banecki, D. Braun, R.W. Glaser, Sequential resonance assignment of medium-sized N-15/C-13- labeled proteins with projected 4D triple resonance NMR experiments, J. Biomol. NMR 11 (1998) 387-405.

[33] N.S. Astrof, C.E. Lyon, R.G. Griffin, Triple resonance solid state NMR experiments with reduced dimensionality evolution periods, J. Magn. Reson. 152 (2001) 303-307.

[34] M. Hong, Solid-state NMR determination of C-13 alpha chemical shift anisotropies for the identification of protein secondary structure, J. Am. Chem. Soc. 122 (2000) 3762-3770.

[35] S. Vijaykumar, C.E. Bugg, W.J. Cook, Structure of ubiquitin refined at $1.8 \AA$ resolution, J. Mol. Biol. 194 (1987) 531-544.

[36] G. Cornilescu, J.L. Marquardt, M. Ottiger, A. Bax, Validation of protein structure from anisotropic carbonyl chemical shifts in a dilute liquid crystalline phase, J. Am. Chem. Soc. 120 (1998) 68366837.

[37] K. Schmidt-Rohr, Complete dipolar decoupling of $13 \mathrm{C}$ and its use in two-dimensional double-quantum solid-state NMR for determining polymer conformations, J. Magn. Reson. Ser. 131 (1998) 209-217.

[38] F.M. Marassi, A simple approach to membrane protein secondary structure and topology based on NMR spectroscopy, Biophys. J. 80 (2001) 994-1003.

[39] A. Lange, S. Luca, M. Baldus, Structural constraints from protonmediated rare-spin correlation spectroscopy, J. Am. Chem. Soc. 124 (2002) 9704-9705.

[40] G. Metz, X.L. Wu, S.O. Smith, Ramped-amplitude cross-polarization in magic-angle-spinning NMR, J. Magn. Reson. Ser. A 110 (1994) 219-227.

[41] S. Hediger, B.H. Meier, N.D. Kurur, G. Bodenhausen, R.R. Ernst, NMR cross-polarization by adiabatic passage through the Hartmann-Hahn condition (APHH), Chem. Phys. Lett. 223 (1994) 283-288.

[42] S.R. Hartmann, E.L. Hahn, Nuclear double resonance in rotating frame, Physical Review 128 (1962) 2042-2053.

[43] A. Pines, M.G. Gibby, J.S. Waugh, Proton-enhanced NMR of dilute spins in solids, J. Chem. Phys. 59 (1973) 569-590.

[44] A.E. Bennett, C.M. Rienstra, M. Auger, K.V. Lakshmi, R.G. Griffin, Heteronuclear decoupling in rotating solids, J. Chem. Phys. 103 (1995) 6951-6958. 